\title{
Spatial Dynamics of A Reaction-Diffusion Model with Distributed Delay
}

\author{
Y. Zhang ${ }^{1}$, X.-Q. Zhao ${ }^{1 *}$ \\ ${ }^{1}$ Department of Mathematics and Statistics \\ Memorial University of Newfoundland \\ St. John's, NL A1C 5S7, Canada
}

\begin{abstract}
This paper is devoted to the study of spreading speeds and traveling waves for a class of reaction-diffusion equations with distributed delay. Such an equation describes growth and diffusion in a population where the individuals enter a quiescent phase exponentially and stay quiescent for some arbitrary time that is given by a probability density function. The existence of the spreading speed and its coincidence with the minimum wave speed of monostable traveling waves are established via the finite-delay approximation approach. We also prove the existence of bistable traveling waves in the case where the associated reaction system admits a bistable structure. Moreover, the global stability and uniqueness of the bistable waves are obtained in the case where the density function has zero tail.
\end{abstract}

Keywords and phrases: Distributed delay, spreading speeds, traveling waves, global stability

Mathematics Subject Classification: 35Q92, 39B72, 92B05, 92D25

\section{Introduction}

In recent years, there have been quite a few research works on biological dynamical systems with quiescent phases, see [5] and [13] for the tumor growth, [10] for the chemostat, [14] for the microbial growth, and [8] for reaction-diffusion equations. For more general mathematical properties of such a class of systems, we refer to $[6,7]$ and references therein. Many researchers have paid attention to spreading speeds and traveling wave solutions for reaction-diffusion equations with time delays (see, e.g., $[2,12,16,18,20-22]$ ). The authors of [2] investigated the existence of the spreading speed and its coincidence with the minimal wave speed for a non-local and time-delayed reaction-diffusion system. In [18] and [12], the authors established the existence, uniqueness and global stability of bistable traveling waves for reaction diffusion equations with finite delays.

Recently, Hadeler and Lutscher [9] proposed the following reaction-diffusion model with distributed delay to describe the evolution of a population in an active phase:

$$
\frac{\partial u}{\partial t}=D \frac{\partial^{2} u}{\partial x^{2}}+p \int_{0}^{+\infty} L(b) u(t-b, x) d b-p u+f(u) .
$$

*Corresponding author. E-mail: zhao@mun.ca 
In this model, $u(t, x)$ is the density of the population in the active phase at time $t$ and location $x$, and $D>0$ is the diffusion coefficient of the population. We assume that the exit time from the active phase is exponentially distributed with parameter $p$, and the exit time from the quiescent phase follows an arbitrary distribution with probability density function $L(b) \geq 0$ satisfying $\int_{0}^{\infty} L(b) d b=1$. Under appropriate conditions, they obtained the minimal wave speed of a traveling wave of (1.1) in the case where the function $f(u)$ admits a monostable structure. The purpose of this paper is to establish the existence of the spreading speed and its coincidence with the minimal wave speed when the function $f(u)$ admits a monostable structure, and the existence and stability of monotone bistable traveling waves when the function $f(u)$ admits a bistable structure. We will appeal to the theory of spreading speeds and traveling waves developed in $[4,11]$ for monotone semiflows, the finite-delay approximation method introduced in [22], and the squeezing technique used in [18].

This paper is organized as follows. In section 2, we establish the existence of the spreading speed and its coincidence with the minimal wave speed in the finite delay case and then extend the results to the infinite delay case. In section 3 , we obtain the existence of monotone bistable traveling waves. At last, in section 4 , we prove the global stability and uniqueness of bistable waves under the assumption that the density function has zero tail.

\section{Spreading speeds and monostable waves}

In this section, we assume that the function $f$ in (1.1) satisfies the following conditions:

$$
f(0)=f(1)=0, f^{\prime}(0)>0>f^{\prime}(1),
$$

and

$$
f(u) \leq f^{\prime}(0) u, f(u)>0 \text { for } u \in(0,1) .
$$

Note that (1.1) admits exactly two constant equilibria $u=0$ and $u=1$ in $[0,1]$. We are interested in the existence of the spreading speed and monostable traveling waves connecting $u=0$ and $u=1$.

Recall that a number $c^{*}>0$ is called the asymptotic spreading speed (in short, spreading speed) for a function $u: \mathbb{R}_{+} \times \mathbb{R}^{n} \rightarrow \mathbb{R}_{+}$with $u(0, x)$ having the compact support if $\lim _{t \rightarrow \infty,|x| \geq c t} u(t, x)=0$ for $c>c^{*}$, and if there exists some $u^{*}>0$ such that $\lim _{t \rightarrow \infty,|x| \leq c t} u(t, x)=u^{*}$ for every $c \in\left(0, c^{*}\right)$.

Let $\mathcal{C}$ be the space of all bounded and continuous functions from $[-\tau, 0] \times \mathbb{R}$ to $\mathbb{R}$ equipped with the compact open topology, and $\mathcal{C}_{+}=\{\psi \in \mathcal{C}: \psi(\theta, x) \geq 0, \forall(\theta, x) \in[-\tau, 0] \times \mathbb{R}\}$. For any $\psi^{1}, \psi^{2} \in \mathcal{C}$, we write $\psi^{2} \geq \psi^{1}$ if $\psi^{2}-\psi^{1} \in \mathcal{C}_{+}$, and $\psi^{2}>\psi^{1}$ if $\psi^{2}-\psi^{1} \in \mathcal{C}_{+} \backslash\{0\}$. For any $a, b, r \in \mathbb{R}$ with $a \leq b$ and $r>0$, we define $\mathcal{C}_{r}:=\{\psi \in \mathcal{C}: r \geq \psi \geq 0\}$ and $[a, b]_{\mathcal{C}}:=\{\psi \in \mathcal{C}: b \geq \psi \geq a\}$. Let $\overline{\mathcal{C}}:=C([-\tau, 0], \mathbb{R})$. Then for any $\bar{\phi} \in \overline{\mathcal{C}}$, we can regard $\bar{\phi}$ as an element in $\mathcal{C}$ by defining $\phi(\theta, x)=\bar{\phi}(\theta), \forall(\theta, x) \in[-\tau, 0] \times \mathbb{R}$. As such, $\overline{\mathcal{C}}$ is a subset of $\mathcal{C}$ with the standard pointwise ordering.

We first consider the following reaction-diffusion equation with finite delay $\tau>0$ :

$$
\frac{\partial u}{\partial t}=D \frac{\partial^{2} u}{\partial x^{2}}+p \int_{0}^{\tau} L(b) u(t-b, x) d b-p u+f(u) .
$$

Define $F: \mathcal{C} \rightarrow C(\mathbb{R}, \mathbb{R})$ and $u_{t} \in \mathcal{C}$ by

$$
F(\phi)(x)=p \int_{0}^{\tau} L(b) \phi(-b, x) d b-p \phi(0, x)+f(\phi(0, x)),
$$

and

$$
u_{t}(\theta, x)=u(t+\theta, x),
$$

for all $(\theta, x) \in[-\tau, 0] \times \mathbb{R}$. Then it is easy to verify $F$ is quasi-monotone on $\overline{\mathcal{C}}$ in the sense that $F(\phi) \leq F(\psi)$ whenever $\phi \leq \psi$ in $\overline{\mathcal{C}}$ and $\phi(0)=\psi(0)$. 
Note that (2.1) can be written as

$$
\frac{\partial u}{\partial t}=D \frac{\partial^{2} u}{\partial x^{2}}+F\left(u_{t}\right), \forall t>0, x \in \mathbb{R} .
$$

By the properties of function $f$, there exists $\tau_{0}>0$ such that for all $\tau \geq \tau_{0}$, the equation

$$
\hat{F}(u):=p u \int_{0}^{\tau} L(b) d b-p u+f(u)=0
$$

admits only two constant solutions 0 and $u_{\tau}^{*}>0$ in $[0,1]$ satisfying

$$
\begin{gathered}
\hat{F}^{\prime}(0)=f^{\prime}(0)-p\left(1-\int_{0}^{\tau} L(b) d b\right)>0, \\
\hat{F}^{\prime}\left(u_{\tau}^{*}\right)=f^{\prime}\left(u_{\tau}^{*}\right)-p\left(1-\int_{0}^{\tau} L(b) d b\right)<0,
\end{gathered}
$$

and $u_{\tau}^{*} \rightarrow 1$ as $\tau \rightarrow \infty$.

Let $X$ be the set of all bounded and continuous functions from $\mathbb{R}$ to $\mathbb{R}$. Clearly, any function in $X$ can be regarded as an element in $\mathcal{C}$. Let $\{T(t)\}_{t \geq 0}$ be the solution semigroup on $X$ associated with the heat equation

$$
\frac{\partial u}{\partial t}=D \Delta u
$$

that is

$$
T(t) \phi(x)=\int_{\mathbb{R}} \frac{e^{\frac{-y^{2}}{4 D t}}}{\sqrt{4 \pi D t}} \phi(x-y) d y, \forall \phi \in X, t>0, x \in \mathbb{R} .
$$

Then system (2.3) can be written as the following integral equation:

$$
u(t, x)=T(t) u(0, \cdot)(x)+\int_{0}^{t} T(t-s) F\left(u_{s}\right)(x) d s, \forall t>0 .
$$

A solution $u(t, x)$ of $(2.5)$ is said to be a mild solution of $(2.3)$, and a function $\bar{u} \in C([-\tau, \infty) \times \mathbb{R}, \mathbb{R})$ is called an upper (a lower) solution of (2.5) if it satisfies

$$
\bar{u}(t, x) \geq(\leq) T(t) \bar{u}(0, \cdot)(x)+\int_{0}^{t} T(t-s) F\left(\bar{u}_{s}\right)(x) d s, \forall t>0, x \in \mathbb{R} .
$$

By a similar argument to that in [2, Lemma 2.1], we have the following result.

Lemma 2.1. For any $\tau \geq \tau_{0}$ and $\phi \in \mathcal{C}_{u_{\tau}^{*}}:=\left\{\phi \in \mathcal{C}: 0 \leq \phi \leq u_{\tau}^{*}\right\}$, system (2.3) has a unique mild solution $u(t, x ; \phi)$ on $[0, \infty)$ and $u(t, x ; \phi)$ is a classic solution of $(2.3)$ for $(t, x) \in[\tau,+\infty) \times \mathbb{R}$. For any pair of upper solution $\bar{u}(t, x)$ and lower solutions $\underline{u}(t, x)$ with $\bar{u}(0, x) \geq \underline{u}(0, x)$, then $\bar{u}(t, x) \geq \underline{u}(t, x)$ holds for all $t \geq 0$ and $x \in \mathbb{R}$.

Let $Q_{t}$ be the solution map of $(2.5)$, that is,

$$
Q_{t}(\phi)(\theta, x)=u(t+\theta, x ; \phi), \forall \theta \in[-\tau, 0], x \in \mathbb{R}, \phi \in C_{u_{\tau}^{*}},
$$

and $\bar{Q}_{t}$ be the restriction of $Q_{t}$ to $\overline{\mathcal{C}}_{u_{\tau}^{*}}$. Then it is easy to verify that $\bar{Q}_{t}$ is the solution semiflow on $\overline{\mathcal{C}}_{u_{\tau}^{*}}$ associated with the following spatially homogeneous delay differential equation

$$
\frac{d u}{d t}=\bar{F}\left(u_{t}\right), \forall t>0
$$


where $\bar{F}$ is the restriction of $F$ to $\overline{\mathcal{C}}_{u_{\tau}^{*}}$. Under the assumption that $\int_{\tau-\epsilon}^{\tau} L(b) d b>0$ for all small $\epsilon>0$, we can verify that $(2.7)$ is cooperative and irreducible in the sense given in [17, Section 5.3]. Thus, $\bar{Q}_{t}$ is eventually strongly monotone on $\overline{\mathcal{C}}_{u_{\tau}^{*}}$. Note that $\bar{F}$ admits only two constant equilibria 0 and $u_{\tau}^{*}$ on $\overline{\mathcal{C}}_{u_{\tau}^{*}}$, and $\hat{F}^{\prime}(0)>0>\hat{F}^{\prime}\left(u_{\tau}^{*}\right)$, which implies that equilibrium 0 is unstable and $u_{\tau}^{*}$ is globally attractive for system (2.7) in $\overline{\mathcal{C}}_{u_{\tau}^{*}} \backslash\{0\}$. By the Dancer-Hess connecting orbit lemma (see, e.g., [23, Section 2.1]), the semiflow $\bar{Q}_{t}$ admits a strongly monotone full orbit connecting 0 and $u_{\tau}^{*}$. It then follows that $Q_{t}$ satisfies all assumptions in [11, Theorem 5.1] for all $t \geq 0$. Then for each $\tau \geq \tau_{0}$, there exists a real number $c_{\tau}^{*}>0$ such that $c_{\tau}^{*}$ is the spreading speed for solutions of $(2.3)$ with initial functions having compact supports for spatial variable $x$, and $c_{\tau}^{*}$ is also the minimal wave speed for monotone traveling waves of (2.3) connecting $u_{\tau}^{*}$ to 0 . To summarize, we have the following result.

Theorem 2.2. Let $\tau \geq \tau_{0}$ be given such that $\int_{\tau-\epsilon}^{\tau} L(b) d b>0$ for all small $\epsilon>0$, and let $u(t, x, \phi, \tau)$ be the unique solution of (2.3) with the initial data $\phi \in \mathcal{C}_{u_{\tau}^{*}}$. Then the following statements are valid:

(1) For any $c>c_{\tau}^{*}$, if $\phi \in \mathcal{C}_{u_{\tau}^{*}}$ with $0 \leq \phi \ll u_{\tau}^{*}$ and $\phi(\cdot, x)=0$ for $x$ outside a bounded interval, then $\lim _{t \rightarrow \infty,|x| \geq c t} u(t, x, \phi, \tau)=0$.

(2) For any $0<c<c_{\tau}^{*}$ and $\sigma \in \overline{\mathcal{C}}_{u_{\tau}^{*}}$ with $\sigma \gg 0$, there is a positive number $r_{\sigma}$ such that if $\phi \in \mathcal{C}_{u_{\tau}^{*}}$ and $\phi(\cdot, x) \gg \sigma$ for $x$ on an interval of length $2 r_{\sigma}$, then $\lim _{t \rightarrow \infty,|x| \leq c t} u(t, x, \phi, \tau)=u_{\tau}^{*}$.

(3) For any $c \geq c_{\tau}^{*}$, (2.3) has a traveling wave solution $U(x-c t)$ such that $U(s)$ is continuous and nonincreasing in $s \in \mathbb{R}$, and $U(+\infty)=0$ and $U(-\infty)=u_{\tau}^{*}$. Moreover, for any $0<c<c_{\tau}^{*}$, (2.3) has no traveling wave $U(x-c t)$ connecting $u_{\tau}^{*}$ to 0 .

In the case where $L(b)=0$ for all sufficiently large $b>0$, system (1.1) reduces to a reaction-diffusion equation with finite delay, and hence, Theorem 2.2 can be used to obtain the spatial dynamics of the reduced system. In the case of infinite delay, since $L(b) \geq 0$ and $\int_{0}^{\infty} L(b) d b>0$, we may choose a sequence $\tau_{n} \rightarrow \infty$ such that $\int_{\tau_{n}-\epsilon}^{\tau_{n}} L(b) d b>0$ for all small $\epsilon>0$ and all $n \geq 1$. Without loss of generality, we assume that $\int_{\tau-\epsilon}^{\tau} L(b) d b>0$ for all small $\epsilon>0$ and all sufficiently large $\tau>0$. Otherwise, we can use $\tau_{n} \rightarrow \infty$ to replace $\tau \rightarrow \infty$ in our arguments for the case of infinite delay.

In order to estimate $c_{\tau}^{*}$, we consider the following linear delay differential equation:

$$
\frac{\partial u}{\partial t}=D \frac{\partial^{2} u}{\partial x^{2}}+p \int_{0}^{\tau} L(b) u(t-b, x) d b-p u+f^{\prime}(0) u .
$$

Note that $f(u) \leq f^{\prime}(0) u$ for all $0 \leq u \leq 1$. Since $f^{\prime}(0)>0$, we see that for any $\epsilon \in(0,1)$, there exists $\delta>0$ such that

$$
f(u) \geq(1-\epsilon) f^{\prime}(0) u, \forall u \in[0, \delta] .
$$

It follows that the condition (F3) in [11, Section 5.1] holds. Let $\left\{M_{t}\right\}_{t \geq 0}$ be the solution semiflow associated with (2.8) and $u(t, x)=e^{-\mu x} v(t), \mu>0$, be a solution of (2.8). Then $v(t)$ satisfies the following delay differential equation:

$$
\frac{d v}{d t}=D \mu^{2} v(t)+p \int_{0}^{\tau} L(b) v(t-b) d b-p v(t)+f^{\prime}(0) v(t) .
$$

Define $B_{\mu}^{t}: \overline{\mathcal{C}} \rightarrow \overline{\mathcal{C}}$ by

$$
B_{\mu}^{t}\left(v_{0}\right)(\theta)=M_{t}\left[e^{-\mu x} v_{0}\right](\theta, 0), \quad \forall v_{0} \in \overline{\mathcal{C}}, \theta \in[-\tau, 0] .
$$

It then follows that $B_{\mu}^{t}$ is the solution map of (2.9) on $\overline{\mathcal{C}}$. Since system (2.9) is cooperative and irreducible (see [17, Section 5.3]), its characteristic equation

$$
\lambda-D \mu^{2}-f^{\prime}(0)+p-p \int_{0}^{\tau} L(b) e^{-\lambda b} d b=0
$$

admits a real root $\lambda_{\tau}(\mu)$, which is greater than the real parts of all other roots (see [17, Theorem 5.5.1]). With the aid of (2.10), it easily follows that there exists $\hat{\tau}>\tau_{0}$ such that $\lambda_{\tau}(\mu)>0$ for all $\tau \geq \hat{\tau}$ and 
$\mu \in(0, \infty)$. Define the function $\Phi_{\tau}(\mu):=\frac{\lambda_{\tau}(\mu)}{\mu}, \forall \mu>0$. In view of the result in [11, Section 5.1], we then have

$$
c_{\tau}^{*}=\inf _{\mu>0} \Phi_{\tau}(\mu) .
$$

By the properties of function $\Phi_{\tau}(\mu)$ as stated in [11, Lemma 3.8], we can verify that there exists a unique $\mu_{\tau}^{*} \in(0,+\infty)$ such that $c_{\tau}^{*}=\Phi_{\tau}\left(\mu_{\tau}^{*}\right)$. From [11, Lemma 3.8(iii)], we see that $\Phi_{\tau}^{\prime}(\mu)$ changes the sign at most once in $(0,+\infty)$. Suppose, by contradiction, that there is an interval $\left[\mu_{1}, \mu_{2}\right] \subset(0,+\infty)$ such that $c_{\tau}^{*}=\Phi_{\tau}(\mu), \forall \mu \in\left[\mu_{1}, \mu_{2}\right]$, that is, $c_{\tau}^{*} \mu=\lambda_{\tau}(\mu), \forall \mu \in\left[\mu_{1}, \mu_{2}\right]$. In view of $(2.10)$, we have

$$
c_{\tau}^{*} \mu=\lambda_{\tau}(\mu)=D \mu^{2}+f^{\prime}(0)-p+p \int_{0}^{\tau} L(b) e^{-\lambda_{\tau}(\mu) b} d b
$$

and hence,

$$
f_{\tau}(\mu):=D \mu^{2}+f^{\prime}(0)-p+p \int_{0}^{\tau} L(b) e^{-\lambda_{\tau}(\mu) b} d b-c_{\tau}^{*} \mu \equiv 0, \forall \mu \in\left[\mu_{1}, \mu_{2}\right] .
$$

On the other hand, we obtain

$$
f_{\tau}^{\prime}(\mu)=2 D \mu-c_{\tau}^{*}-p c_{\tau}^{*} \int_{0}^{\tau} L(b) e^{-c_{\tau}^{*} \mu b} b d b, \quad \forall \mu \in\left[\mu_{1}, \mu_{2}\right] .
$$

It is easy to observe that $f_{\tau}^{\prime}(\mu)$ is strictly increasing in $\left[\mu_{1}, \mu_{2}\right]$, which contradicts $f_{\tau}(\mu) \equiv 0$ for all $\mu \in\left[\mu_{1}, \mu_{2}\right]$. Therefore, there is a unique $u_{\tau}^{*}>0$ such that $\left(\mu_{\tau}^{*}, c_{\tau}^{*}\right)$ satisfies $c_{\tau}^{*}=\Phi_{\tau}\left(\mu_{\tau}^{*}\right)$ and $\left.\frac{\partial \Phi_{\tau}(\mu)}{\partial \mu}\right|_{\mu_{\mu}^{*}}=0$. Let

$$
P_{\tau}(c, \lambda)=D \mu^{2}-c \mu-p+f^{\prime}(0)+p \int_{0}^{\tau} L(b) e^{-\lambda b c} d b .
$$

Then $\left(\mu_{\tau}^{*}, c_{\tau}^{*}\right)$ can be uniquely determined by

$$
P_{\tau}(c, \mu)=0 \text { and } \frac{\partial P_{\tau}(c, \mu)}{\partial \mu}=0
$$

Now we show that for any given $\mu>0, \lambda_{\tau}(\mu)$ is nondecreasing in $\tau \in[\hat{\tau}, \infty)$. Suppose, by contradiction, that there exist some $\mu_{0}>0$ and $\tau_{2}>\tau_{1} \geq \hat{\tau}$ such that $\lambda_{\tau_{2}}\left(\mu_{0}\right)<\lambda_{\tau_{1}}\left(\mu_{0}\right)$. Then we see from (2.10) that

$$
\begin{aligned}
\lambda_{\tau_{2}}\left(\mu_{0}\right)-\lambda_{\tau_{1}}\left(\mu_{0}\right) & =p \int_{0}^{\tau_{2}} L(b) e^{-\lambda_{\tau_{2}}\left(\mu_{0}\right) b} d b-p \int_{0}^{\tau_{1}} L(b) e^{-\lambda_{\tau_{1}}\left(\mu_{0}\right) b} d b \\
& \geq p \int_{0}^{\tau_{1}} L(b)\left(e^{-\lambda_{\tau_{2}}\left(\mu_{0}\right) b}-e^{-\lambda_{\tau_{1}}\left(\mu_{0}\right) b}\right) d b \geq 0,
\end{aligned}
$$

which is a contradiction. Further, (2.10) implies that

$$
\lambda_{\tau}(\mu) \leq D \mu^{2}+f^{\prime}(0) .
$$

Therefore, $\lambda(\mu):=\lim _{\tau \rightarrow \infty} \lambda_{\tau}(\mu)<+\infty$ exists.

Consider the following equation:

$$
\lambda=D \mu^{2}+f^{\prime}(0)-p+p \int_{0}^{\infty} L(b) e^{-\lambda b} d b
$$

We claim that for each $\mu>0, \lambda(\mu)$ is a unique real root of (2.11), and the real parts of all other roots of (2.11) are less than $\lambda(\mu)$. Indeed, $\lambda_{\tau}(\mu)$ satisfies

$$
\lambda_{\tau}(\mu)=D \mu^{2}+f^{\prime}(0)-p+p \int_{0}^{\tau} L(b) e^{-\lambda_{\tau}(\mu) b} d b
$$


and $\lambda_{\tau}(\mu)>0$ when $\tau$ is large enough. Letting $\tau \rightarrow \infty$ in the above equation, we see that $\lambda(\mu)$ is a real root of (2.11). For the sake of contradiction, suppose that there are some $\mu_{0}>0$ and $a \neq \lambda\left(\mu_{0}\right)$ such that $a$ is another real root of (2.11). Without loss of generality, we assume that $a>\lambda\left(\mu_{0}\right)\left(a<\lambda\left(\mu_{0}\right)\right)$. Then we obtain

$$
a-\lambda\left(\mu_{0}\right)=p \int_{0}^{\infty} L(b)\left(e^{-a b}-e^{-\lambda\left(\mu_{0}\right) b}\right) d b<0(>0),
$$

which is a contradiction. Thus, $\lambda(\mu)$ is a unique real root of (2.11). Similarly, if we assume, by contradiction, that $\alpha+i \beta$ is a complex root of $(2.11)$ with $\alpha \geq \lambda\left(\mu_{0}\right)$, then we have

$$
0 \leq \alpha-\lambda\left(\mu_{0}\right)=p \int_{0}^{\infty} L(b)\left(e^{-\alpha b} \cos (\beta b)-e^{-\lambda\left(\mu_{0}\right) b}\right) d b<0,
$$

a contradiction.

Since $\lambda(\mu) \geq \lambda_{\tau}(\mu), \forall \tau \geq \hat{\tau}$, we see from (2.11) and (2.12) that

$$
\begin{aligned}
0 & \leq \lambda(\mu)-\lambda_{\tau}(\mu) \\
& =p \int_{\tau}^{\infty} L(b) e^{-\lambda(\mu) b} d b+p \int_{0}^{\tau} L(b)\left(e^{-\lambda(\mu) b}-e^{-\lambda_{\tau}(\mu) b}\right) d b \\
& \leq p \int_{\tau}^{\infty} L(b) d b, \forall \tau \geq \hat{\tau}, \mu \in(0, \infty) .
\end{aligned}
$$

This implies that $\lim _{\tau \rightarrow \infty} \lambda_{\tau}(\mu)=\lambda(\mu)$ uniformly for $\mu \in(0, \infty)$. Note that

$$
\left|\lambda(\mu)-\lambda\left(\mu_{0}\right)\right| \leq\left|\lambda(\mu)-\lambda_{\tau}(\mu)\right|+\left|\lambda_{\tau}(\mu)-\lambda_{\tau}\left(\mu_{0}\right)\right|+\left|\lambda_{\tau}\left(\mu_{0}\right)-\lambda\left(\mu_{0}\right)\right|
$$

holds for all $\tau \geq \hat{\tau}, \mu, \mu_{0} \in(0, \infty)$. It then follows that $\lambda(\mu)$ is a continuous function on $(0, \infty)$, and hence, the function $\Phi(\mu):=\frac{\lambda(\mu)}{\mu}$ is continuous in $\mu \in(0, \infty)$. In view of $(2.11)$ and the properties of $\Phi(\mu)$, it is easy to verify that $\Phi(+0)=\Phi(+\infty)=+\infty$. Thus, $c^{*}:=\inf _{\mu>0} \Phi(\mu)$ can be obtained at some finite value $\mu^{*}$. Combing the properties of $\Phi_{\tau}(\mu)$, we see that $\left(c^{*}, \mu^{*}\right)$ is a unique solution to $c^{*}=\Phi\left(\mu^{*}\right)$ and $\left.\frac{d \Phi(\mu)}{d \mu}\right|_{\mu=\mu^{*}}=0$. Let

$$
P(c, \mu)=D \mu^{2}-c \mu-p+f^{\prime}(0)+p \int_{0}^{\infty} L(b) e^{-\lambda b c} d b .
$$

Then we can easily show that the following result holds true.

Lemma 2.3. The following statements are valid:

(1) For any $c>c^{*}$, there is $\mu>0$ such that $P(c, \mu)<0$,

(2) $\left(c^{*}, \mu^{*}\right)$ is uniquely determined by $P(c, \mu)=0$ and $\frac{\partial P(c, \mu)}{\partial \mu}=0$,

(3) $c_{\tau}^{*}$ is nondecreasing in $\tau \in[\hat{\tau}, \infty)$, and $\lim _{\tau \rightarrow \infty}\left(c_{\tau}^{*}, \mu_{\tau}^{*}\right)=\left(c^{*}, \mu^{*}\right)$.

Now we consider system (1.1) with infinite time delay. By the argument similar to that in [2, Lemma 2.8.], we have the following result on the existence and uniqueness of solutions of (1.1).

Lemma 2.4. For any $\phi \in C((-\infty, 0] \times \mathbb{R},[0,1])$, system $(1.1)$ has a unique mild solution $u(t, x ; \phi)$ with $u_{0}(\phi)=\phi$ and $u_{t}(\phi) \in C((-\infty, 0] \times \mathbb{R},[0,1])$ for all $t \geq 0$.

Consider the linearized equation of system (1.1) at the zero solution:

$$
\frac{\partial u}{\partial t}=D \frac{\partial^{2} u}{\partial x^{2}}+p \int_{0}^{+\infty} L(b) u(t-b, x) d b-p u+f^{\prime}(0) u .
$$

In a similar way to (2.6), we can define the upper and lower solutions for (2.13). Then we have the following comparison principle. We omit the proof here since it is essentially the same as that of $[2$, Lemma 2.9.]. 
Lemma 2.5. Let $\bar{u}(t, x)$ and $\underline{u}(t, x)$ be the upper and lower solutions of $(2.13)$. If $\bar{u}_{0} \geq \underline{u}_{0}$, then $\bar{u}(t, x) \geq$ $\underline{u}(t, x), \forall t \geq 0, x \in \mathbb{R}$.

We are now in a position to prove the existence of the spreading speed for model (1.1).

Theorem 2.6. Let $\phi \in C((-\infty, 0] \times \mathbb{R},[0,1])$ and $u(t, x ; \phi)$ be the solution of $(1.1)$ with $u_{0}=\phi$. Then the following statements are valid:

(1) For any $c>c^{*}$, if $\phi(\cdot, x)=0$ for $x$ outside a bounded interval, then

$$
\lim _{t \rightarrow \infty|x| \geq c t} u(t, x ; \phi)=0 .
$$

(2) For any $0<c<c^{*}$ and $\sigma \in \overline{\mathcal{C}}_{1}$ with $\sigma \gg 0$, there is a positive number $r_{\sigma}$ such that if $\phi \in \mathcal{C}_{1}$ and $\phi(\cdot, x) \gg \sigma$ for $x$ on an interval of length $2 r_{\sigma}$, then

$$
\lim _{t \rightarrow \infty,|x| \leq c t} u(t, x ; \phi)=1 .
$$

Proof. We use the arguments similar to those in [19, Proposition 2.2] and [22, Theorem 2.1]. In the case where $c>c^{*}$, let $\phi(\theta, x)$ be given as in statement (1). For fixed $\bar{c} \in\left(c^{*}, c\right)$ and $\bar{\lambda}>0$ such that $P(\bar{c}, \bar{\lambda})<0$, there exists a large positive number $M>0$ such that

$$
\phi(t, x) \leq M e^{\bar{\lambda}(\bar{c} t-z x)}, \forall(t, x) \in(-\infty, 0] \times \mathbb{R}, z=1 \text { or } z=-1 .
$$

For $z=1$ or -1 , the function $\bar{u}(t, x):=M e^{\bar{\lambda}(\bar{c} t-z x)}$ satisfies

$$
\begin{aligned}
& \frac{\partial \bar{u}(t, x)}{\partial t}-D \bar{u}_{x x}-p \int_{0}^{+\infty} L(b) \bar{u}(t-b, x) d b+\left(p-f^{\prime}(0)\right) \bar{u}(t, x) \\
= & M e^{\bar{\lambda}(\bar{c} t-z x)}(-P(\bar{c}, \bar{\lambda}))>0 .
\end{aligned}
$$

That is, $\bar{u}(t, x)$ is an upper solution of (2.13). Since $f(u) \leq f^{\prime}(0) u, u(t, x ; \phi)$ is a lower solution of $(2.13)$. Letting $z=\frac{x}{|x|}, x \neq 0$. By the comparison principle in Lemma 2.5, we obtain

$$
u(t, x ; \phi) \leq \bar{u}(t, x)=M e^{\bar{\lambda}(\bar{c} t-|x|)} \quad \forall(t, x) \in[0,+\infty) \times \mathbb{R},
$$

which implies that $\lim _{t \rightarrow \infty,|x| \geq c t} u(t, x ; \phi)=0$.

In the case where $c \in\left(0, c^{*}\right)$, since $\lim _{\tau \rightarrow+\infty} c_{\tau}^{*}=c^{*}$, there exists $\tau_{1}>\tau_{0}$ such that $c_{\tau}^{*}>c$ for all $\tau>\tau_{1}$. For any given $\tau>\tau_{1}$, we define

$$
\hat{\phi}(\theta, x)=\min \left\{\phi(\theta, x), u_{\tau}^{*}\right\}, \forall(\theta, x) \in[-\tau, 0] \times \mathbb{R} .
$$

Let $u(t, x, \tau ; \hat{\phi})$ be the solution of $(2.1)$ with $u_{0}=\hat{\phi}$. Note that $u(t, x ; \phi)$ is an upper solution of $(2.1)$. By the comparison principle in Lemma 2.1, we have

$$
u(t, x ; \phi) \geq u(t, x, \tau ; \hat{\phi}), \forall(t, x) \in[0,+\infty) \times \mathbb{R} .
$$

This, together with Theorem 2.2 (2), implies that

$$
1 \geq \limsup _{t \rightarrow \infty,|x| \leq c t} u(t, x ; \phi) \geq \liminf _{t \rightarrow \infty,|x| \leq c t} u(t, x ; \phi) \geq \liminf _{t \rightarrow \infty,|x| \leq c t} u(t, x, \tau ; \hat{\phi})=u_{\tau}^{*}, \forall \tau>\tau_{1} .
$$

Letting $\tau \rightarrow+\infty$, we then have $\lim _{t \rightarrow \infty,|x| \leq c t} u(t, x ; \phi)=1$.

The following results show that the spreading speed $c^{*}$ obtained above is also the minimum wave speed for monotone traveling waves of system (1.1). 
Theorem 2.7. The following statements are valid:

(1) For any $c \geq c^{*}$, system (1.1) has a traveling wave solution $U(x-c t)$ connecting 1 to 0 , and $U(\xi)$ is continuous and nonincreasing in $\xi \in \mathbb{R}$.

(2) For any $0<c<c^{*}$, system (1.1) has no traveling wave solution $U(x-c t)$ connecting 1 to 0 .

Proof. For any given $c>c^{*}$, we choose a sequence $\tau_{n} \rightarrow \infty$ such that $c_{\tau_{n}}^{*}<c$ and $\lim _{n \rightarrow \infty} c_{\tau_{n}}^{*}=c^{*}$. By Theorem $2.2(3)$, system (2.1) with $\tau=\tau_{n}$ admits a traveling wave $U_{n}(x-c t)$ such that $U_{n}(-\infty)=u_{\tau_{n}}^{*}$ and $U_{n}(+\infty)=0$. Thus, we obtain

$$
\begin{aligned}
J_{n}(U)(\xi):= & D U^{\prime \prime}(\xi)+c U^{\prime}(\xi)-p U(\xi)+f(U(\xi))+p \int_{0}^{\tau_{n}} L(b) U(\xi+c b) d b \\
= & D U^{\prime \prime}(\xi)+c U^{\prime}(\xi)-\alpha U(\xi)+\alpha U(\xi)-p U(\xi)+f(U(\xi)) \\
& +p \int_{0}^{\tau_{n}} L(b) U(\xi+c b) d b=0
\end{aligned}
$$

where $\alpha>0$ is a fixed large number such that the function $\alpha u-p u+f(u)$ is nondecreasing in $u \in[0,1]$. Define the operator

$$
H_{n}(U)(\xi):=\alpha U(\xi)-p U(\xi)+f(U(\xi))+p \int_{0}^{\tau_{n}} L(b) U(\xi+c b) d b .
$$

Clearly, $\left\{H_{n}(U)(\xi)\right\}_{n=1}^{\infty}$ is uniformly bounded when $U(\xi)$ is bounded. It then follows that

$$
\begin{aligned}
U(\xi)= & k_{1} e^{\lambda_{1} \xi}+k_{2} e^{\lambda_{2} \xi} \\
& +\frac{1}{D\left(\lambda_{2}-\lambda_{1}\right)}\left(\int_{-\infty}^{\xi} e^{\lambda_{1}(\xi-\eta)} H_{n}(U)(\eta) d \eta+\int_{\xi}^{+\infty} e^{\lambda_{2}(\xi-\eta)} H_{n}(U)(\eta) d \eta\right),
\end{aligned}
$$

where $k_{1}, k_{2}$ are arbitrary constants and

$$
\lambda_{1}=\frac{-c-\sqrt{c^{2}+4 D \alpha}}{2 D}<0, \quad \lambda_{2}=\frac{-c+\sqrt{c^{2}+4 D \alpha}}{2 D}>0 .
$$

Since $U_{n}(\xi)$ satisfies (2.15) and is bounded, it follows that $U_{n}(\xi)$ satisfies $(2.17)$ with $k_{1}=k_{2}=0$, that is,

$$
U_{n}(\xi)=\frac{1}{D\left(\lambda_{2}-\lambda_{1}\right)}\left(\int_{-\infty}^{\xi} e^{\lambda_{1}(\xi-\eta)} H_{n}\left(U_{n}\right)(\eta) d \eta+\int_{\xi}^{+\infty} e^{\lambda_{2}(\xi-\eta)} H_{n}\left(U_{n}\right)(\eta) d \eta\right)
$$

Thus, the sequences $U_{n}^{\prime}(\xi), U_{n}^{\prime \prime}(\xi)$ and $U_{n}^{\prime \prime \prime}(\xi)$ are uniformly bounded for all $n \geq 1$ due to the uniform boundedness of $U_{n}(\xi)$ and $H_{n}\left(U_{n}\right)(\xi)$. By the spatial translation invariance of the original equation, we can assume $U_{n}(0)=\frac{1}{2}$. By the Arizela-Ascoli theorem and a diagonal procedure, it follows that $\left\{U_{n}(\xi), U_{n}^{\prime}(\xi), U_{n}^{\prime \prime}(\xi)\right\}$ has a convergent subsequence, which is convergent uniformly on each compact set in $\mathbb{R}$. Without loss of generality, denote $U_{n}(\xi) \rightarrow U^{*}(\xi)$. Then we have

$$
\left(U_{n}(\xi), U_{n}^{\prime}(\xi), U_{n}^{\prime \prime}(\xi)\right) \rightarrow\left(U^{*}(\xi), U^{* \prime}(\xi), U^{* \prime \prime}(\xi)\right) .
$$

Define

$$
J(U)(\xi):=D U^{\prime \prime}(\xi)+C U^{\prime}(\xi)-p U(\xi)+f(U(\xi))+p \int_{0}^{+\infty} L(b) U(\xi-c b) d b .
$$

Then $\lim _{n \rightarrow \infty} J_{n}\left(U_{n}\right)(\xi)=J\left(U^{*}\right)(\xi)$ pointwise, which implies that $U^{*}(\xi)$ is a solution of $J(U)(\xi)=0$. Since $U_{n}(\xi)$ is nonincreasing and $U_{n}(-\infty)=u_{n}^{*}, U_{n}(\infty)=0, U^{*}(\xi)$ is nonincreasing and bounded. Therefore $U^{*}(+\infty)$ exists and satisfies the following equation

$$
-p x+f(x)+p \int_{0}^{\infty} L(b) d b=f(x)=0 .
$$


Then we have

$$
U^{*}(-\infty)=1>\frac{1}{2}=U^{*}(0)>U^{*}(+\infty)=0 .
$$

Therefore, $U^{*}(\xi)$ is a traveling wave solution of (1.1).

For $c=c^{*}$, by the same limiting argument as in [22, Theorem 3.1], we can obtain the existence of monotone traveling wave $U\left(x-c^{*} t\right)$ connecting 1 to 0 . The nonexistence of traveling wave can be proven by the contradiction argument as in [22, Theorem 3.1].

\section{Existence of bistable waves}

In this section, we consider the case where function $f$ has three zero points $0<x^{*}<1$ on $[0,1]$ satisfying

$$
f^{\prime}(0)<0, f^{\prime}\left(x^{*}\right)>0 \text { and } f^{\prime}(1)<0 .
$$

We establish the existence of the wavefronts connecting two stable equilibria 0 and 1.

We choose $\tau^{*}$ large enough such that for any $\tau \geq \tau^{*}$, the delay equation

$$
\frac{\partial u}{\partial t}=D \frac{\partial^{2} u}{\partial x^{2}}+p \int_{0}^{\tau} L(b) u(t-b, x) d b-p u+f(u)
$$

admits only three constant equilibria $0<\alpha_{\tau}<\beta_{\tau}$ on $[0,1]$ satisfying $\lim _{\tau \rightarrow \infty} \alpha_{\tau}=x^{*}$ and $\lim _{\tau \rightarrow \infty} \beta_{\tau}=1$, and $\hat{F}$, as defined in $(2.4)$, satisfies $\hat{F}^{\prime}(0)<0, \hat{F}^{\prime}\left(\alpha_{\tau}\right)>0$, and $\hat{F}^{\prime}\left(\beta_{\tau}\right)<0$. We first establish the existence of the nondecreasing bistable traveling waves of system (3.1) connecting 0 and $\beta_{\tau}$.

Define function $\bar{F}$ on $\mathcal{C}$ by $\bar{F}(\phi)=p \int_{0}^{\tau} L(b) \phi(-b) d b-p \phi(0)+f(\phi(0))$. Then we have

$$
\begin{aligned}
D \bar{F}(\phi) h & =\left(f^{\prime}(\phi(0))-p\right) h(0)+p \int_{0}^{\tau} h(-b) L(b) d b \\
& =\left(f^{\prime}(\phi(0))-p\right) h(0)+p \int_{-\tau}^{0} h(b) L(-b) d b \\
& =\left(f^{\prime}(\phi(0))-p\right) h(0)+p \int_{-\tau}^{0} h(b) d \eta(\phi),
\end{aligned}
$$

where $\eta(\phi)$ is a positive Borel measure on $[-\tau, 0]$ defined as $\eta(\phi) \Omega=\int_{\Omega} L(-b) d b$ for any measurable subset $\Omega \subset[-\tau, 0]$. Since $L(b) \geq 0$ and $\int_{0}^{\infty} L(b) d b>0$, without loss of generality, we assume that $\tau$ is chosen such that $\eta(\phi)[-\tau,-\tau+\epsilon]=\int_{-\tau}^{-\tau+\epsilon} L(-b) d b>0$ for all small $\epsilon>0$. Thus, assumption (E4) in [4, Section 6.4] holds.

For any $\epsilon>0$, define a linear operator $L_{\epsilon}: \mathcal{C}_{\beta_{\tau}} \rightarrow C(\mathbb{R}, \mathbb{R})$ by

$$
L_{\epsilon} \phi=p \int_{0}^{\tau} L(b) \phi(-b, x) d b-p \phi(0, x)+(1-\epsilon) f^{\prime}\left(\alpha_{\tau}\right) \phi(0, x) .
$$

Then $L_{\epsilon} \phi \rightarrow D F\left(\alpha_{\tau}\right) \phi$ as $\epsilon \rightarrow 0$, where the operator $D F\left(\alpha_{\tau}\right)$ is defined by

$$
D F\left(\alpha_{\tau}\right) \phi=p \int_{0}^{\tau} L(b) \phi(-b, x) d b-p \phi(0, x)+f^{\prime}\left(\alpha_{\tau}\right) \phi(0, x) .
$$

It is easy to verify that there exists $\delta \in\left(0, \beta_{\tau}\right)$ such that

$$
F\left(\alpha_{\tau}+\phi\right) \geq L_{\epsilon}(\phi) \text { and } F\left(\alpha_{\tau}-\phi\right) \leq-L_{\epsilon}(\phi), \forall \phi \in \mathcal{C}_{\delta},
$$

that is, assumption (E5) in [4] holds. Further, assumptions (E1)-(E3) in [4] are also satisfied. By [4, Theorem 6.4], we then have the following result. 
Theorem 3.1. Let $\tau \geq \tau^{*}$ be given such that $\int_{\tau-\epsilon}^{\tau} L(b) d b>0$ for all small $\epsilon>0$. Then system (3.1) admits a nondecreasing traveling wave $V_{\tau}\left(x-c_{\tau} t\right)$ with $V_{\tau}(-\infty)=0$ and $V_{\tau}(+\infty)=\beta_{\tau}$.

In order to prove the boundedness of $\left\{c_{\tau}\right\}_{\tau \geq \tau^{*}}$, we use the similar ideas to those in [3] to construct upper and lower solutions. Choose an increasing function $\rho \in C^{2}(\mathbb{R}, \mathbb{R})$ such that

$$
\begin{aligned}
& \rho(\xi)=0, \forall \xi \leq 0 ; \quad \rho^{\prime}(\xi) \in(0,1), \forall \xi \in(0,4) ; \\
& \rho(\xi)=1, \quad \forall \xi \geq 4 ; \quad\left|\rho^{\prime \prime}(\xi)\right| \leq 1, \quad \forall \xi \in(0,4) .
\end{aligned}
$$

Then we have the following result.

Lemma 3.2. Define

$$
\begin{aligned}
& \underline{v}(t, x)=v_{-}(x-c t ; \delta, \sigma):=\rho(\sigma(x-c t))-\delta, \\
& \bar{v}(t, x)=v_{+}(x+c t ; \delta, \sigma):=\rho(\sigma(x+c t))+\delta .
\end{aligned}
$$

Then there exist $\bar{\delta}>0, \bar{\sigma}>0$ and $\bar{c}>0$ such that for any $\delta \in[\bar{\delta} / 2, \bar{\delta}], \sigma \in[\bar{\sigma} / 2, \bar{\sigma}]$ and $c \geq \bar{c}, \underline{v}(t, x)$ and $\bar{v}(t, x)$ are a lower solution and an upper solution of (3.1) with $\tau>\tau_{0}$, respectively.

Proof. Define $F_{\infty}: \mathbb{R} \rightarrow \mathbb{R}$ by

$$
F_{\infty}(x):=p x \int_{0}^{\infty} L(b) d b-p x+f(x)
$$

Then $F_{\infty}^{\prime}(0)=f^{\prime}(0)<0, F_{\infty}^{\prime}\left(x^{*}\right)=f^{\prime}\left(x^{*}\right)>0$ and $F_{\infty}^{\prime}(1)=f^{\prime}(1)<0$. By the continuous differentiability of $F_{\infty}$, there exists $0<\bar{\delta}<1$ such that for all $\delta \in[0, \bar{\delta}]$, the following inequalities hold:

$$
\begin{gathered}
F_{\infty}(-\delta) \geq-F_{\infty}^{\prime}(0) \delta+\frac{1}{2} f^{\prime}(0) \delta=-\frac{1}{2} f^{\prime}(0) \delta>0, \\
F_{\infty}(1-\delta) \geq-F_{\infty}^{\prime}(1) \delta+\frac{1}{2} f^{\prime}(1) \delta=-\frac{1}{2} f^{\prime}(0) \delta>0 .
\end{gathered}
$$

Thus, we can find $\theta_{0}>0, \bar{\sigma}>0$ such that for any $\theta \in\left[0, \theta_{0}\right], \sigma \in[0, \bar{\sigma}]$ and $\delta \in\left[\frac{\bar{\delta}}{2}, \bar{\delta}\right]$, the following two inequalities hold:

$$
\begin{gathered}
F_{\infty}(\theta-\delta)>D \sigma^{2}+p \int_{\tau^{*}}^{\infty} L(b) d b \\
F_{\infty}((1-\theta)-\delta)>D \sigma^{2}+p \int_{\tau^{*}}^{\infty} L(b) d b,
\end{gathered}
$$

where we may choose $\tau^{*}$ large enough if it is necessary.

Letting $\xi=x-c t$, we then have

$$
\begin{aligned}
& D \frac{\partial^{2} \underline{\underline{v}}}{\partial x^{2}}-\frac{\partial \underline{v}}{\partial t}+F\left(\underline{v}_{t}\right)(x) \\
= & D v_{-}^{\prime \prime}(x-c t)+c v_{-}^{\prime}(x-c t)+F\left(\underline{v}_{t}\right)(x) \\
= & D v_{-}^{\prime \prime}(\xi)+c v_{-}^{\prime}(\xi)-p v_{-}(\xi)+f\left(v_{-}(\xi)\right)+p \int_{0}^{\tau} L(b)(\rho(\sigma(\xi+c b))-\delta) d b \\
\geq & D \sigma^{2} \rho^{\prime \prime}(\sigma \xi)+c \sigma \rho^{\prime}(\sigma \xi)-p v_{-}(\xi)+f\left(v_{-}(\xi)\right)+p \int_{0}^{\tau} L(b)(\rho(\sigma(\xi))-\delta) d b \\
= & D \sigma^{2} \rho^{\prime \prime}(\sigma \xi)+c \sigma \rho^{\prime}(\sigma \xi)+F_{\infty}\left(v_{-}(\xi)\right)-p v_{-}(\xi) \int_{\tau}^{\infty} L(b) d b \\
\geq & D \sigma^{2} \rho^{\prime \prime}(\sigma \xi)+c \sigma \rho^{\prime}(\sigma \xi)+F_{\infty}\left(v_{-}(\xi)\right)-p \int_{\tau^{*}}^{\infty} L(b) d b
\end{aligned}
$$


Denote $m:=\min _{\rho(\xi) \in\left[\theta_{0}, 1-\theta_{0}\right]} \rho^{\prime}(\xi)>0$ and $F_{\min }:=\min _{-1 \leq x \leq 1} F_{\infty}(x)$. Let $\bar{c}>0$ sufficiently large such that

$$
c \sigma m \geq-F_{\min }+D \sigma^{2}+p \int_{\tau^{*}}^{\infty} L(b) d b, \forall c>\bar{c} .
$$

If $\rho(\sigma \xi) \in\left[0, \theta_{0}\right] \cup\left[1-\theta_{0}, 1\right]$, then

$$
D \frac{\partial^{2} \underline{v}}{\partial x^{2}}-\frac{\partial \underline{v}}{\partial t}+F\left(\underline{v}_{t}\right)(x) \geq-D \sigma^{2}+D \sigma^{2}+p \int_{\tau^{*}}^{\infty} L(b) d b-p \int_{\tau^{*}}^{\infty} L(b) d b=0 ;
$$

If $\rho(\sigma \xi) \in\left[\theta_{0}, 1-\theta_{0}\right]$, then

$$
D \frac{\partial^{2} \underline{v}}{\partial x^{2}}-\frac{\partial \underline{v}}{\partial t}+F\left(\underline{v}_{t}\right)(x) \geq-D \sigma^{2}+c \sigma m+F_{\min }-p \int_{\tau^{*}}^{\infty} L(b) d b \geq 0 .
$$

Consequently, $\underline{v}(t, x)$ is a lower solution of (3.1). Similarly, we can prove that $\bar{v}(t, x)$ is an upper solution of (3.1).

Lemma 3.3. $\left\{c_{\tau}\right\}_{\tau \geq \tau^{*}}$ is bounded.

Proof. By Lemma 3.2, we see that there exist $\bar{c}, \bar{\delta}$ and $\bar{\sigma}$ independent of $\tau \geq \tau^{*}$ such that $v_{-}(x-\bar{c} t ; \bar{\delta}, \overline{\bar{\sigma}})$ and $v_{+}(x+\bar{c} t ; \bar{\delta}, \bar{\sigma})$ are a lower and an upper solution of system (3.1), respectively. Note that when $\tau^{*}$ is large enough, the following inequalities hold:

$$
\begin{gathered}
v_{-}(-\infty ; \bar{\delta}, \bar{\sigma})=-\bar{\delta}<0=V_{\tau}(-\infty), \\
v_{-}(+\infty ; \bar{\delta}, \bar{\sigma})=1-\bar{\delta}<\beta_{\tau}=V_{\tau}(+\infty)
\end{gathered}
$$

Since $v_{-}$and $V_{\tau}$ are all nondecreasing functions, there exists $\xi_{\tau} \in \mathbb{R}$ such that

$$
V_{\tau}\left(\xi+\xi_{\tau}\right) \geq v_{-}(\xi ; \bar{\delta}, \bar{\sigma}), \forall \xi \in \mathbb{R} .
$$

By the comparison principle, we then have

$$
V_{\tau}\left(x-c_{\tau} t+\xi_{\tau}\right) \geq v_{-}(x-\bar{c} t ; \bar{\delta}, \bar{\sigma}), \forall t \geq 0, x \in \mathbb{R} .
$$

Thus, we obtain

$$
V_{\tau}\left(\cdot+\left(\bar{c}-c_{\tau}\right) t+\xi_{\tau}\right) \geq v_{-}(\cdot ; \bar{\delta}, \bar{\sigma}), \forall t \geq 0,
$$

which implies that $c_{\tau} \leq \bar{c}, \forall \tau \geq \tau_{0}$. Suppose, by contradiction, that $c_{\tau}>\bar{c}$. Then we have $0=$ $V_{\tau}(-\infty) \gg 0$, which is a contradiction. Similarly, we can prove that $c_{\tau} \geq-\bar{c}$. Therefore, we have $\left|c_{\tau}\right| \leq \bar{c}$ for all $\tau>\tau^{*}$.

Lemma 3.4. The following statements are valid:

(1) If $U(x+c t)$ is a nondecreasing traveling wave of $(1.1)$ with $U(-\infty)=x^{*}$ and $U(+\infty)=1$, then $c>0$.

(2) If $U(x+c t)$ is a nondecreasing traveling wave of (1.1) with $U(-\infty)=0$ and $U(+\infty)=x^{*}$, then $c<0$.

Proof. Let $U(x+c t)$ be a nondecreasing traveling waves in (1), by the spatial symmetry of (1.1). Then $W(x-c t):=U(-x+c t)=U(-(x-c t))$ is a nonincreasing traveling wave of $(1.1)$ with $W(-\infty)=1$ and $W(+\infty)=x^{*}$. By the analysis in Section 2, we have the similar results as in Theorem 2.7 for system (1.1) restricted on $\left[x^{*}, 1\right]$, that is, there is a positive number $c_{1}$ such that $c>c_{1}>0$.

If $U(x+c t)$ is a nondecreasing traveling waves in (2), then $\bar{W}(x+c t)=x^{*}-U(x+c t)$ is a nonincreasing traveling wave of the following system:

$$
\frac{\partial u}{\partial t}=D \frac{\partial^{2} u}{\partial x^{2}}+p \int_{0}^{+\infty} L(b) u(t-b, x) d b-p u-f\left(x^{*}-u\right)
$$

with $\bar{W}(-\infty)=x^{*}$ and $\bar{W}(+\infty)=0$. Similarly, for system (3.4) restricted on $\left[0, x^{*}\right]$, there is a positive number $c_{0}>0$ such that $-c>c_{0}>0$, which implies that $c<0$. 
Now we are in a position to prove the main result of this section.

Theorem 3.5. System (1.1) admits a nondecreasing bistable traveling wave $U(x-c t)$ with $U(-\infty)=0$ and $U(+\infty)=1$.

Proof. From Lemma 3.3, we see that $\left\{c_{\tau}\right\}_{\tau>\tau^{*}}$ is bounded. Then there is a sequence of real numbers $n_{k}>$ $\tau^{*}, k \in \mathbb{N}$ such that $c_{n_{k}}$ converges to some real number $c$ as $n_{k} \rightarrow+\infty$. In view of Theorem 3.1, system (3.1) with $\tau=n_{k}$ has a wavefront $\left(U_{n_{k}}, c_{n_{k}}\right)$ such that $U_{n_{k}}$ is a monotone function with $U_{n_{k}}(-\infty)=0$ and $U_{n_{k}}(+\infty)=1$. Thus, there exist $\xi_{k}, \eta_{k} \in \mathbb{R}$ such that $U_{n_{k}}\left(\xi_{k}\right)=\frac{x^{*}}{2}$ and $U_{n_{k}}\left(\eta_{k}\right)=\frac{1+x^{*}}{2}$. Let

$$
V_{k}(\cdot)=U_{n_{k}}\left(\cdot+\xi_{k}\right) \text { and } W_{k}(\cdot)=U_{n_{k}}\left(\cdot+\eta_{k}\right) .
$$

Then $V_{k}(0)=\frac{x^{*}}{2}$ and $W_{k}(0)=\frac{1+x^{*}}{2}$ for all $k \geq 1$. Note that $\left\{V_{k}\right\}_{k \geq 1}$ and $\left\{W_{k}\right\}_{k \geq 1}$ are monotone function sequences with $V_{k}(-\infty)=W_{k}(-\infty)=0$ and $V_{k}(+\infty)=W_{k}(+\infty)=1$. By Helly's theorem, it follows that there exist subsequences, still denoted as $\left\{V_{k}\right\}_{k \geq 1}$ and $\left\{W_{k}\right\}_{k \geq 1}$, and monotone functions $V$ and $W$ such that $\lim _{k \rightarrow \infty} V_{k}=V$ and $\lim _{k \rightarrow} W_{k}=W$ pointwise on $\mathbb{R}$ as $k \rightarrow+\infty, V(0)=\frac{x^{*}}{2}$, and $W(0)=\frac{1+x^{*}}{2}$. Denote $V_{ \pm}(\cdot)=V(\cdot \pm 0)$ and $W_{ \pm}(\cdot)=W(\cdot \pm 0)$. Then $V_{-}$and $W_{-}$are left-continuous, while $V_{+}$and $W_{+}$are right-continuous. Note that $V_{ \pm}(\xi)=V(\xi)$ and $W_{ \pm}(\xi)=W(\xi)$ almost everywhere on $\mathbb{R}$.

Next we show that both $(V, c)$ and $(W, c)$ are traveling wavefronts of $(1.1)$. Note that $V_{k}$ satisfies the following system:

$$
D V_{k}^{\prime \prime}(\xi)+c_{n_{k}} V_{k}^{\prime}(\xi)-p V_{k}(\xi)+p \int_{0}^{n_{k}} L(b) V_{k}(\xi+c b) d b+f\left(V_{k}(\xi)\right)=0,
$$

which is equivalent to the following integral equation

$$
\begin{aligned}
V_{k}(\xi)= & \frac{1}{\sqrt{c_{n_{k}}^{2}+4 D p}}\left\{\int _ { - \infty } ^ { \xi } e ^ { \lambda _ { 1 k } ( \xi - \eta ) } \left(p \int_{0}^{n_{k}} L(b) V_{k}(\eta+c b) d b+f\left(V_{k}(\eta)\right) d \eta\right.\right. \\
& +\int_{\xi}^{+\infty} e^{\lambda_{2 k}(\xi-\eta)}\left(p \int_{0}^{n_{k}} L(b) V_{k}(\eta+c b) d b+f\left(V_{k}(\eta)\right) d \eta\right\}
\end{aligned}
$$

where $\lambda_{1 k}=\frac{-c_{n_{k}}-\sqrt{c_{n_{k}}^{2}+4 D p}}{2 D}<0$ and $\lambda_{2 k}=\frac{-c_{n_{k}}+\sqrt{c_{n_{k}}^{2}+4 D p}}{2 D}>0$. Further,

$$
\begin{aligned}
& \lambda_{1}=\lim _{n \rightarrow \infty} \lambda_{1 k}=\frac{-c-\sqrt{c^{2}+4 D p}}{2 D}, \\
& \lambda_{2}=\lim _{n \rightarrow \infty} \lambda_{2 k}=\frac{-c+\sqrt{c^{2}+4 D p}}{2 D} .
\end{aligned}
$$

By the Lebesgue dominated convergence theorem, it then follows that

$$
\begin{aligned}
V(\xi)= & \frac{1}{\sqrt{c^{2}+4 D p}}\left\{\int _ { - \infty } ^ { \xi } e ^ { \lambda _ { 1 } ( \xi - \eta ) } \left(p \int_{0}^{+\infty} L(b) V(\eta+c b) d b+f(V(\eta)) d \eta\right.\right. \\
& +\int_{\xi}^{+\infty} e^{\lambda_{2}(\xi-\eta)}\left(p \int_{0}^{+\infty} L(b) V(\eta+c b) d b+f(V(\eta)) d \eta\right\}
\end{aligned}
$$

which is equivalent to

$$
D V^{\prime \prime}(\xi)+c V^{\prime}(\xi)-p V(\xi)+p \int_{0}^{\infty} L(b) V(\xi+c b) d b+f(V(\xi))=0,
$$

that is, $V(x-c t)$ satisfies equation (1.1). Similarly, we can prove that $W(x-c t)$ also satisfies equation (1.1). 
Now we need to verify the boundary conditions. It is obvious that $V( \pm \infty)$ and $W( \pm \infty)$ exist from the monotonicity of $V$ and $W$. Note that $V( \pm \infty)$ and $W( \pm \infty)$ are the zero points of $f(u)=0$. Since $V(0)=\frac{x^{*}}{2}$ and $W(0)=\frac{1+x^{*}}{2}$, it follows that $V(-\infty)=0$ and $V(+\infty)=x^{*}$ or 1 , and $W(-\infty)=x^{*}$ or 0 , and $W(+\infty)=1$. Note that $W$ and $V$ are all traveling wave solutions of system (1.1). By Lemma 3.4, we see that $V(+\infty)=x^{*}$ and $W(-\infty)=x^{*}$ cannot happen simultaneously. Thus, either $(V, c)$ or $(W, c)$ is a wavefront of (1.1) connecting 0 and 1 .

\section{Global stability of bistable waves}

In this section, we prove the global asymptotic stability with shift and uniqueness of monotone bistable traveling waves. Note that it is much more difficult to analyze the global stability of traveling waves for an infinite delay model. So we assume that there exists $\tau>0$ such that $L(b) \equiv 0$ for all $b \geq \tau$. Accordingly, system (1.1) becomes the following differential equation with finite distributed delay:

$$
\frac{\partial u}{\partial t}=D \frac{\partial^{2} u}{\partial x^{2}}+p \int_{0}^{\tau} L(b) u(t-b, x) d b-p u+f(u) .
$$

Let $u(t, x)=U(x-c t)$ be the monotone bistable traveling wave solution of (4.1), as obtained in the Section 3. Then we have $U^{\prime}(\xi) \geq 0$ and $U^{\prime}(\xi) \not \equiv 0$ for all $\xi \in \mathbb{R}$. Let $v(t, x)=\frac{\partial u(t, x)}{x}$. Then $v(t, x)$ satisfies the following equation:

$$
\frac{\partial v}{\partial t}=D \frac{\partial^{2} v}{\partial x^{2}}+p \int_{0}^{\tau} L(b) v(t-b, x) d b-p v+f^{\prime}(u) v .
$$

By the strong maximum principle, it follows that $v(t, x)>0$ for all $t>0$, and hence, $U^{\prime}(\xi)>0$ for all $\xi \in \mathbb{R}$.

Lemma 4.1. Let $U(x-c t)$ be a strictly monotone bistable traveling wave solution of (4.1). Then there exist positive numbers $\beta_{0}, \sigma_{0}$, and $\bar{\delta}$ such that for any $\delta \in(0, \bar{\delta}]$ and every $\zeta_{0} \in \mathbb{R}$, the functions $w^{ \pm}$ defined by

$$
w^{ \pm}(t, x):=U\left(x-c t+\zeta_{0} \pm \sigma_{0} \delta\left[1-e^{-\beta_{0} t}\right]\right) \pm \delta e^{-\beta_{0} t}
$$

are an upper solution and a lower solutions of $(4.1)$ on $[0,+\infty)$, respectively.

Proof. Without loss of generality, we assume $\zeta_{0}=0$. Note that $f(0)=f(1)=0, f^{\prime}(0)<0$, and $f^{\prime}(1)<0$. Then there exist $L_{1}>0$ and $\delta^{*} \in(0,1)$ such that

$$
\begin{gathered}
f(x)>0, x \in\left[-\delta^{*}, 0\right) \text { and } f^{\prime}(x)<-L_{1}, x \in\left[-\delta^{*}, \delta^{*}\right] \\
f(x)>0, x \in\left(1, \delta^{*}\right] \text { and } f^{\prime}(x)<-L_{1}, x \in\left[1-\delta^{*}, 1+\delta^{*}\right] .
\end{gathered}
$$

Since $U(-\infty)=0$ and $U(+\infty)=1$, there exists $M>0$ such that

$$
U(\xi) \in\left[-\frac{\delta^{*}}{2}, \frac{\delta^{*}}{2}\right], \xi<-M \text { and } U(\xi) \in\left[1-\frac{\delta^{*}}{2}, 1+\frac{\delta^{*}}{2}\right], \xi>M
$$

Let $\bar{\delta}=\frac{\delta^{*}}{2}, L_{2}=\min _{\xi \in[-M, M]} U^{\prime}(\xi)>0$ and $L_{3}=\max _{x \in[0,1+\bar{\delta}]} f(x)>0$. Fix $\beta_{0}>0$ small such that $\beta_{0}+p\left(\int_{0}^{\tau} L(b) e^{\beta_{0} b} d b-1\right)<L_{1}$ and $\sigma_{0}=\frac{2\left(L_{1}+L_{3}\right)}{L_{2} \beta_{0}}>0$. It is easy to verify that function $U(\xi)$ satisfies

$$
D U^{\prime \prime}(\xi)+c U^{\prime}(\xi)+p \int_{0}^{\tau} L(b) U(\xi+c b) d b-p U(\xi)+f(U(\xi))=0, \forall \xi \in \mathbb{R}
$$


Denote $\xi(t):=x-c t+\sigma_{0} \delta\left(1-e^{-\beta_{0} t}\right)$. Then for any $t \geq 0$ and $\delta \in(0, \bar{\delta})$, we have

$$
\begin{aligned}
& \frac{\partial w^{+}(x, t)}{\partial t}-D \frac{\partial^{2} w^{+}(x, t)}{\partial x^{2}}-F\left(w^{+}(t, x)\right) \\
= & U^{\prime}(\xi)\left[-c+\sigma_{0} \delta \beta_{0} e^{-\beta_{0} t}\right]-\delta \beta_{0} e^{-\beta_{0} t}-D U^{\prime \prime}(\xi)+p U(\xi)+p \delta e^{-\beta_{0} t} \\
& -p \int_{0}^{\tau} L(b)\left[U\left(x-c t+c b+\sigma_{0} \delta\left(1-e^{-\beta_{0}(t-b)}\right)\right)+\delta e^{-\beta_{0}(t-b)}\right] d b-f\left(U(\xi)+\delta e^{-\beta_{0} t}\right) \\
= & p \int_{0}^{\tau} L(b)\left[U(\xi+c b)-U\left(\xi+c b+\sigma_{0} \delta e^{-\beta_{0} t}\left(1-e^{\beta_{0} b}\right)\right)\right] d b-p \delta e^{-\beta_{0} t} \int_{0}^{\tau} L(b) e^{\beta_{0} b} d b \\
& +\sigma_{0} \delta \beta_{0} e^{-\beta_{0} t} U^{\prime}(\xi)-\delta \beta_{0} e^{-\beta_{0} t}+p \delta e^{-\beta_{0} t}+f(U(\xi))-f\left(U(\xi)+\delta e^{-\beta_{0} t}\right) \\
\geq & \delta e^{-\beta_{0} t}\left[-p \int_{0}^{\tau} L(b) e^{\beta_{0} b} d b+\sigma_{0} \beta_{0} U^{\prime}(\xi)-\beta_{0}+p-f^{\prime}\left(U(\xi)+\theta \delta e^{-\beta_{0} t}\right)\right] \\
= & \delta e^{-\beta_{0} t}\left[\sigma_{0} \beta_{0} U^{\prime}(\xi)-\beta_{0}-p\left(\int_{0}^{\tau} L(b) e^{\beta_{0} b} d b-1\right)-f^{\prime}\left(U(\xi)+\theta \delta e^{-\beta_{0} t}\right)\right], \quad \theta \in(0,1)
\end{aligned}
$$

In the case where $|\xi(t)|>M$, by the choice of $\bar{\delta}, \beta_{0}$ and $\theta \in(0,1)$, we have

$$
U(\xi)+\theta \delta e^{-\beta_{0} t} \in\left[0, \delta^{*}\right] \text { or } U(\xi)+\theta \delta e^{-\beta_{0} t} \in\left[1,1+\delta^{*}\right]
$$

which implies that $\left.f^{\prime}\left(U(\xi)+\theta \delta e^{-\beta_{0} t}\right)\right)<-L_{1}$. It then follows that

$$
\frac{\partial w^{+}(x, t)}{\partial t}-D \frac{\partial^{2} w^{+}(x, t)}{\partial x^{2}}-F\left(w^{+}(t, x)\right) \geq \delta e^{-\beta_{0} t}\left[-\beta_{0}-p\left(\int_{0}^{\tau} L(b) e^{\beta_{0} b} d b-1\right)+L_{1}\right] \geq 0 ;
$$

In the case where $|\xi(t)| \leq M$, we have

$$
\frac{\partial w^{+}(x, t)}{\partial t}-D \frac{\partial^{2} w^{+}(x, t)}{\partial x^{2}}-F\left(w^{+}(t, x)\right) \geq \delta e^{-\beta_{0} t}\left[\sigma_{0} \beta_{0} L_{2}-L_{1}-L_{3}\right]=L_{1}+L_{3}>0 .
$$

Similarly, we can verify $w^{-}$is a lower solution of system (4.1).

Let $\tilde{\delta}=\min \left\{\frac{x^{*}}{2}, \frac{1-x^{*}}{2}, \delta^{*}\right\}$, and $\rho(\cdot) \in C^{2}(\mathbb{R}, \mathbb{R})$ be the function defined in Section 3 . Then we have the following result.

Lemma 4.2. For any $\delta \in(0, \tilde{\delta}]$, there exist two positive number $\epsilon$ and $C$ such that for any $\xi \in R$, the functions $v^{+}$and $v^{-}$defined by

$$
\begin{aligned}
& v^{+}(t, x)=(1+\delta)-\left[1-\left(x^{*}-2 \delta\right) e^{-\epsilon t}\right] \rho(-\epsilon(x-\xi+C t)), \\
& v^{-}(t, x)=-\delta+\left[1-\left(1-x^{*}-2 \delta\right) e^{-\epsilon t}\right] \rho(\epsilon(x-\xi-C t)),
\end{aligned}
$$

are an upper solution and a lower solutions of system (4.1) on $[0, \infty)$, respectively.

Proof. Without loss of generality, we take $\xi=0$. We first verify that $v^{-}$is a lower solution. For any $\delta \in(0, \tilde{\delta}]$, we choose $\varepsilon=\varepsilon(\delta)>0$ small enough such that the following three inequalities hold:

$$
\begin{gathered}
\left(1-x^{*}\right) e^{\varepsilon \tau}<1, \\
-D \varepsilon^{2}-\varepsilon-p \tau \varepsilon+\min _{u \in\left[-\delta,-\frac{\delta}{2}\right]} f(u)>0,
\end{gathered}
$$

and

$$
-D \varepsilon^{2}-\varepsilon-p \tau \varepsilon+\min _{u \in\left[x^{*}+\frac{\delta}{2}, 1-\delta\right]} f(u)>0 .
$$


It is easy to verify that $v^{-}(x, t) \in[-\delta, 1-\delta]$ for all $x \in R$ and $t \geq-\tau$. Choose $C=C(\delta)>0$ large enough such that

$$
-D \varepsilon^{2}-\varepsilon+\varepsilon C\left[1-\left(1-x^{*}\right) e^{\varepsilon \tau}\right] \cdot \min _{\rho(s) \in\left[\frac{\delta}{2}, 1-\frac{\delta}{2}\right]} \rho^{\prime}(s)-p \tau \varepsilon+\min _{u \in[-\delta, 1-\delta]} f(u)>0 .
$$

Denote $\zeta=\varepsilon(x-C t)$. Then for any $t \geq-\tau$, the following inequalities hold:

$$
\begin{aligned}
\frac{\partial v^{-}(t, x)}{\partial t} & =-C \varepsilon\left[1-\left(1-x^{*}-2 \delta\right) e^{-\varepsilon t}\right] \rho^{\prime}(\zeta)+\varepsilon\left(1-x^{*}-2 \delta\right) e^{-\varepsilon t} \rho(\zeta) \\
& \leq-C \varepsilon\left[1-\left(1-x^{*}-2 \delta\right) e^{-\varepsilon \tau}\right] \rho^{\prime}(\zeta)+\varepsilon\left(1-x^{*}-2 \delta\right) e^{-\varepsilon \tau} \rho(\zeta) \\
& \leq-C \varepsilon\left[1-\left(1-x^{*}\right) e^{-\varepsilon \tau}\right] \rho^{\prime}(\zeta)+\varepsilon\left(1-x^{*}\right) e^{-\varepsilon \tau} \rho(\zeta) \\
& \leq \varepsilon
\end{aligned}
$$

It then follows that

$$
\begin{aligned}
& D \frac{\partial v^{-}(t, x)}{\partial x^{2}}-\frac{\partial v^{-}(t, x)}{\partial t}+F\left(v^{-}(t, x)\right) \\
= & D\left[1-\left(1-x^{*}-2 \delta\right) e^{-\varepsilon t}\right] \varepsilon^{2} \rho^{\prime \prime}(\zeta)+C \varepsilon\left[1-\left(1-x^{*}-2 \delta\right) e^{-\varepsilon t}\right] \rho^{\prime}(\zeta) \\
& -\varepsilon\left(1-x^{*}-2 \delta\right) e^{-\varepsilon t} \rho(\zeta)+p \int_{0}^{\tau} L(b) v^{-}(x, t-b) d b-p v^{-}(x, t)+f\left(v^{-}(x, t)\right) \\
\geq & -D \varepsilon^{2}+C \varepsilon\left[1-\left(1-x^{*}\right) e^{\varepsilon \tau}\right] \rho^{\prime}(\zeta)-\varepsilon+p \int_{0}^{\tau} L(b) v^{-}(x, t-b) d b \\
& -p v^{-}(x, t)+f\left(v^{-}(x, t)\right), \quad \forall t \geq 0 .
\end{aligned}
$$

Then we discuss (4.4) in the following three cases.

Case (i) If $\rho(\zeta)<\frac{\delta}{2}$, it is easy to verify that $v^{-}(x, t) \in\left[-\delta,-\frac{\delta}{2}\right]$ for all $x \in \mathbb{R}$ and $t \geq-\tau$. Then we have

$$
\begin{aligned}
& D \frac{\partial v^{-}(t, x)}{\partial x^{2}}-\frac{\partial v^{-}(t, x)}{\partial t}+F\left(v^{-}(t, x)\right. \\
\geq & -D \varepsilon^{2}-\varepsilon+p \int_{0}^{\tau} L(b) v^{-}(x, t-b) d b-p v^{-}(x, t)+f\left(v^{-}(x, t)\right) \\
\geq & -D \varepsilon^{2}-\varepsilon+p \min _{s \in[t-\tau, t]} v^{-}(x, s)-p v^{-}(x, t)+\min _{u \in\left[-\delta,-\frac{\delta}{2}\right]} f(u) \\
= & -D \varepsilon^{2}-\varepsilon+p\left(v^{-}\left(x, t^{*}\right)-v^{-}(x, t)\right)+\min _{u \in\left[-\delta,-\frac{\delta}{2}\right]} f(u), \quad t^{*} \in[t-\tau, t] \\
\geq & -D \varepsilon^{2}-\varepsilon+p\left(t^{*}-t\right) \max _{s \in\left[t^{*}, t\right]} \frac{\partial v^{-}(x, s)}{\partial s}+\min _{u \in\left[-\delta,-\frac{\delta}{2}\right]} f(u) \\
\geq & -D \varepsilon^{2}-\varepsilon+p\left(t^{*}-t\right) \varepsilon+\min _{u \in\left[-\delta,-\frac{\delta}{2}\right]} f(u) \\
\geq & -D \varepsilon^{2}-\varepsilon-p \tau \varepsilon+\min _{u \in\left[-\delta,-\frac{\delta}{2}\right]} f(u)>0 .
\end{aligned}
$$
get

Case (ii) If $\rho(\zeta)>1-\frac{\delta}{2}$, it is easy to verify that $v^{-}(x, t) \in\left[x^{*}+\frac{\delta}{2}, 1-\delta\right]$. By a similar analysis, we

$$
\begin{gathered}
D \frac{\partial v^{-}(t, x)}{\partial x^{2}}-\frac{\partial v^{-}(t, x)}{\partial t}+F\left(v^{-}(t, x)\right. \\
\geq-D \varepsilon^{2}-\varepsilon-p \tau \varepsilon+\min _{u \in\left[x^{*}+\frac{\delta}{2}, 1-\delta\right]} f(u)>0 .
\end{gathered}
$$


Case (iii) If $\rho(\zeta) \in\left[\frac{\delta}{2}, 1-\frac{\delta}{2}\right]$, we have

$$
\begin{aligned}
& D \frac{\partial v^{-}(t, x)}{\partial x^{2}}-\frac{\partial v^{-}(t, x)}{\partial t}+F\left(v^{-}(t, x)\right) \\
\geq & -D \varepsilon^{2}+C \varepsilon\left[1-\left(1-x^{*}\right) e^{\varepsilon \tau}\right] \rho^{\prime}(\zeta)-\varepsilon-p \tau \varepsilon+\min _{u \in[-\delta, 1-\delta]} f(u) \\
\geq & -D \varepsilon^{2}+C \varepsilon\left[1-\left(1-x^{*}\right) e^{\varepsilon \tau}\right] \min _{\rho(\zeta) \in\left[\frac{\delta}{2}, 1-\frac{\delta}{2}\right]} \rho^{\prime}(\zeta)-\varepsilon-p \tau \varepsilon+\min _{u \in[-\delta, 1-\delta]} f(u) \\
> & 0 .
\end{aligned}
$$

Therefore, $v^{-}$is a lower solution of (4.1). Similarly, we can verify that $v^{+}$is an upper solution of (4.1) on $[0, \infty)$.

In view of Lemma 4.1 , for any $\eta \in \mathbb{R}$ and $\delta \in[0, \infty)$, we define $w^{ \pm}(t, x, \eta, \delta)$ by

$$
w^{ \pm}(t, x, \eta, \delta)=U\left(x-c t+\eta \pm \sigma_{0} \delta\left(1-e^{-\beta_{0} t}\right)\right) \pm \delta e^{-\beta_{0} t}, \forall x \in \mathbb{R}, \quad t \in[-\tau, \infty),
$$

where $\sigma_{0}$ and $\beta_{0}$ are as in Lemma 4.1 and $\beta_{0}$ is chosen small such that $3 e^{\beta_{0} \tau}<4$. Thanks to Lemmas 4.1 and 4.2 , we can do similar analysis to that in [18] to obtain the following result. The details of the proof are omitted here since they are essentially the same as those in [18, Lemma 3.2].

Lemma 4.3. Let $U(x-c t)$ be a monotone traveling wave of the system $(4.1)$, and $\varphi \in[0,1]_{\mathcal{C}}$ be given such that

$$
\begin{aligned}
& \liminf _{x \rightarrow \infty} \min _{s \in[-\tau, 0]} \varphi(x, s)>x^{*} \\
& \limsup _{x \rightarrow-\infty} \max _{s \in[-\tau, 0]} \varphi(x, s)<x^{*} .
\end{aligned}
$$

Then for any $\delta>0$, there exists $T>0, \xi \in \mathbb{R}$ and $h>0$ such that

$$
w_{0}^{-}(x,-c T+\xi, \delta)(s) \leq u_{T}(x, \varphi)(s) \leq w_{0}^{+}(x,-c T+\xi+h, \delta)(s)
$$

for all $s \in[-\tau, 0]$ and $x \in \mathbb{R}$.

By Lemma 2.1, system (4.1) admits the comparison principle. In order to get the global stability of traveling waves, we need the following stronger comparison principle.

Lemma 4.4. For any pair of upper solution $\bar{u}(x, t)$ and lower solutions $\underline{u}(t, x)$ of system $(4.1)$ on $[0, \infty)$ with $-\delta^{*} \leq \underline{u}(t, x), \bar{u}(x, x t) \leq 1+\delta^{*}$. If $\bar{u}(s, x) \geq \underline{u}(s, x)$ for all $s \in[-\tau, 0]$, then there exists a function $\theta(J, t) \in C([0, \infty) \times(0, \infty), \mathbb{R})$ such that the following inequalities hold:

$$
\bar{u}(t, x)-\underline{u}(t, x) \geq \theta\left(J, t-t_{0}\right) \int_{z}^{z+1}\left(\bar{u}\left(t_{0}, y\right)-\underline{u}\left(t_{0}, y\right)\right) d y
$$

for all $t \geq 0$ and $x \in \mathbb{R}$.

Proof. Let $\tilde{u}(t, x)=\bar{u}(t, x)-\underline{u}(t, x)$. Then Lemma 2.1 implies that $\tilde{u}(t, x) \geq 0$ for all $t \in[-\tau, \infty)$ and $x \in \mathbb{R}$. For any given $t_{0}>0$, by (2.5) and definition of upper and lower solutions, we have

$$
\begin{aligned}
\tilde{u}(t, x) & \geq T\left(t-t_{0}\right) \tilde{u}\left(t_{0}, \cdot\right)(x)+\int_{t_{0}}^{t} T(t-r)\left(F\left(\underline{u}_{r}\right)(x)-F\left(\bar{u}_{r}\right)\right)(x) d r \\
& \geq T\left(t-t_{0}\right) \tilde{u}\left(\left(t_{0}, \cdot\right)\right)(x)+\int_{t_{0}}^{t} T(t-r)[-p \tilde{u}(r, x)+f(\bar{u}(r, x))-f(\underline{u}(r, x))] d r \\
& \geq T\left(t-t_{0}\right) \tilde{u}\left(\left(t_{0}, \cdot\right)\right)(x)-L^{*} \int_{t_{0}}^{t} T(t-r) \tilde{u}(r, x) d r
\end{aligned}
$$


where $L^{*}=p+\max _{u \in\left[-\delta^{*}, 1+\delta^{*}\right]} f^{\prime}(u)>0$. Let

$$
z(t, x)=e^{-L^{*}\left(t-t_{0}\right)} T\left(t-t_{0}\right) \tilde{u}\left(t_{0}, x\right), t \geq t_{0} .
$$

Then $z(t, x)$ is the solution of the following reaction-diffusion equation:

$$
\frac{\partial u(t, x)}{\partial t}=D \frac{\partial^{2} u(t, x)}{\partial x^{2}}-L^{*} u(t, x)
$$

Thus,

$$
\left.z(t, x)=T\left(t-t_{0}\right) z\left(t_{0}, x\right)-L^{*} \int_{t_{0}}^{t} T(t-r) z(r, x)\right) d r, t \geq t_{0} .
$$

By [15, Proposition 3], it follows that $\tilde{u}(t, x) \geq z(t, x)$ for all $t \geq t_{0}$ and $x \in \mathbb{R}$. Define the function $\theta(J, t) \in C([0, \infty) \times(0, \infty), \mathbb{R})$ by

$$
\theta(J, t)=\frac{1}{\sqrt{4 D \pi t}} \exp \left(-L^{*} t-\frac{(J+1)^{2}}{4 D t}\right), J \geq 0, t>0 .
$$

Then we obtain

$$
\begin{aligned}
\tilde{u}(t, x) & \geq e^{-L^{*}\left(t-t_{0}\right)} T\left(t-t_{0}\right) \tilde{u}\left(t_{0}, x\right) \\
& \geq \frac{e^{-L^{*}\left(t-t_{0}\right)}}{\sqrt{4 D \pi\left(t-t_{0}\right)}} \int_{-\infty}^{\infty} \exp \left(-\frac{(x-y)^{2}}{4 D\left(t-t_{0}\right)}\right) \tilde{u}\left(t_{0}, y\right) d y \\
& \geq \frac{e^{-L^{*}\left(t-t_{0}\right)}}{\sqrt{4 D \pi\left(t-t_{0}\right)}} \int_{z}^{z+1} \exp \left(-\frac{(x-y)^{2}}{4 D\left(t-t_{0}\right)}\right) \tilde{u}\left(t_{0}, y\right) d y \\
& \geq \theta\left(J, t-t_{0}\right) \int_{z}^{z+1} \tilde{u}\left(t_{0}, y\right) d y
\end{aligned}
$$

for all $x \in \mathbb{R}$ with $|x-z| \leq J$ and $t>t_{0} \geq 0$.

By the stronger comparison principle in Lemma 4.4, we have the following result, whose proof is omitted here since it is similar to that of [18, Lemma 3.1].

Lemma 4.5. Let $U(x-c t)$ be a monotone traveling wave solution of system (4.1). Then there exists a positive number $\epsilon^{*}$ such that if $u(t, x)$ is a solution of $(4.1)$ on $[0, \infty)$ with $0 \leq u(t, x) \leq 1$ for $x \in \mathbb{R}$ and $t \in[0, \infty)$, and (4.7) holds for some $\xi \in \mathbb{R}, h>0, \delta \in\left(0, \min \left(\bar{\delta}, \frac{1}{\sigma_{0}}\right)\right)$ and $T>0$, then for any $t \geq T+\tau+1$, there exist $\hat{\xi}(t), \hat{\delta}(t)$, and $\hat{h}(t)$ such that

$$
w_{0}^{-}(x,-c t+\hat{\xi}(t), \hat{\delta}(t))(s) \leq u_{t}(x, \varphi)(s) \leq w_{0}^{+}(x,-c t+\hat{\xi}(t)+\hat{h}(t), \hat{\delta}(t))(s)
$$

for all $s \in[-\tau, 0], x \in \mathbb{R}$, and $\hat{\xi}(t), \hat{\delta}(t), \hat{h}(t)$ satisfying

$$
\begin{aligned}
& \hat{\xi}(t) \in\left[\xi-\sigma_{0} \delta-2 \sigma_{0}\left(\delta+\epsilon^{*} \min (h, 1)\right) e^{\beta_{0} \tau}, \xi+h+\sigma_{0} \delta\right], \\
& \hat{\delta}(t)=\left(\delta e^{-\beta_{0}}+\epsilon^{*} \min (h, 1)\right) e^{-\beta_{0}(t-(T+\tau+1))}, \\
& \hat{h}(t) \in\left[0, h+\left(3 e^{\beta_{0} \tau}-4\right) \sigma_{0} \epsilon^{*} \min (h, 1)+3 e^{\beta_{0} \tau} \sigma_{0} \delta\right] .
\end{aligned}
$$

By using Lemmas 4.3 and 4.5, the squeezing technique, which was introduced in [1], and the arguments similar to those in [18, Theorems 3.3-3.4], we can prove the following result on the global stability and uniqueness of bistable waves for system (4.1). 
Theorem 4.6. Let $U(x-c t)$ be a monotone traveling wave solution of system (4.1). Then $U(x-c t)$ is globally asymptotically stable with phase shift in the sense that there exists $k>0$ such that for any $\psi \in[0,1]_{\mathcal{C}}$ satisfying (4.5) and (4.6), the solution $u(t, x, \psi)$ of (4.1) satisfies

$$
|u(t, x, \psi)-U(x-c t+\xi)| \leq K e^{-k t}, \quad \forall x \in \mathbb{R}, t \geq 0
$$

for some $K=K(\psi)>0$ and $\xi=\xi(\psi) \in \mathbb{R}$. Moreover, $U(x-c t)$ is unique up to a translation in the sense that for any traveling wave solution $\bar{U}(x-\bar{c} t)$ with $0 \leq \bar{U}(\xi) \leq 1, \xi \in \mathbb{R}$, we have $\bar{c}=c$ and $\bar{U}(\cdot)=U\left(\xi_{0}+\cdot\right)$ for some $\xi_{0} \in \mathbb{R}$.

Acknowledgements. We would like to thank two anonymous referees for their valuable comments and suggestions, which led to an improvement of our original manuscript.

\section{References}

[1] X. Chen. Existence, uniqueness and asymptotic stability of traveling waves in nonlocal evolution equations. Adv. Diff. Eq., 2(1997), 125-160.

[2] J. Fang, J. Wei, X.-Q. Zhao. Spatial dynamics of a nonlocal and time-delayed reaction-diffusion system. J. Diff. Eq., 245(2008), 2749-2770.

[3] J. Fang, X.-Q. Zhao. Monotone wavefronts for partially degenerate reaction-diffusion systems. J. Dyn. Diff. Eq., 21(2009), 663-680.

[4] J. Fang, X.-Q. Zhao. Bistable traveling waves for monotone semiflows with applications. Journal of European Math. Soc., in press.

[5] M. Gyllenberg, G. F. Webb. A nonlinear structured population model of tumor growth with quiescence. J. Math. Biol., 28(1990), 671-694.

[6] K. P. Hadeler. Quiescent phases and stability. Lin. Alg. Appl. , 428(2008), 1620-1627.

[7] K. P. Hadeler. Homogenerous systems with a quiescent phase. Math. Models Natur. Phenom. , 3(2008), $115-125$.

[8] K. P. Hadeler, M. A. Lewis. Spatial dynamics of the diffusive logistic equation with a sedentary compartment. Can. Appl. Math. Q. , 10(2002), 473-499.

[9] K. P. Hadeler, F. Lutscher. Quiescent phases with distributed exit times. Disc. Cont. Dyn. Sys.(Ser. B), 17(2012), 849-869.

[10] W. Jäger, S. Krömker, B. Tang. Quiescence and transient growth dynamics in chemostat models. Math. Biosci. , 119(1994), 225-239.

[11] X. Liang, X.-Q. Zhao. Asymptotic speeds of spread and traveling waves for monotone semiflow with applications. Comm. Pure Appl. Math., 60(2007), 1-40, Erratum: Comm. Pure Appl. Math., 61(2008), 137-138.

[12] S. Ma, J. Wu. Existence, uniqueness and asymptotic stability of traveling wavefronts in A non-local delayed diffusion equation. J. Dyn. Diff. Eq., 19(2007), 391-436.

[13] A. Maler, F. Lutscher. Cell cycle times and the tumor control probability. Mathematics in Medicine and Biology, $27(2010), 313-342$.

[14] T. Malik, H. Smith. A resource-based model of microbial quiescence. J. Math. Biol., 53(2006), $231-252$.

[15] R. H. Martin, H. L. Smith. Abstract functional differential equations and reaction-diffusion systems. Trans. Amer. Math. Soc., 321(1990), 1-44.

[16] K. W. Schaaf. Asymptotic behavior and traveling wave solutions for parabolic functional-differential equations Trans. Amer. Math. Soc., 302(1987), 587-615.

[17] H. L. Smith. Monotone dynamical systems: An introduction to the Theory of Competitive and Cooperative Systems. Math. Surveys Monogr.,vol. 41. Amer. Math. Soc., Providence, RI, 1995.

[18] H. L. Smith, X.-Q. Zhao. Global asymptotic stability of traveling waves in delayed reaction-diffusion equations SIAM J. Math. Anal., 31(2000), 514-534.

[19] H. R. Thieme, X.-Q. Zhao. Asymptotic speeds of spread and traveling waves for integral equations and delayed reactiondiffusion models. J. Diff. Eq, 195(2003), 430-470.

[20] Z.-C. Wang, W.-T. Li, S. Ruan. Travelling wave fronts in reaction-diffusion systems with spatio-temporal delays. J. Diff. Eq, 222(2006), 185-232.

[21] J. Wu, X. Zou. Traveling wave fronts of reaction-diffusion systems with delay. J. Dyn. Diff. Eq., 13(2001), $651-687$. Erratum: J. Dyn. Diff. Eq., 20(2008), 531-533.

[22] X.-Q. Zhao, D. Xiao. The asymptotic speed of spread and traveling waves for a vector disease model. J. Dyn. Diff. Eq., 18(2006), 1001-1019, Erratum: J. Dyn. Diff. Eq., 20(2008), 277-279.

[23] X.-Q. Zhao. Dynamical Systems in Population Biology. Springer-Verlag, New York, 2003. 